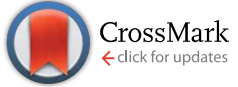

Cite this: RSC Adv., 2015, 5, 11604

Received 16th October 2014

Accepted 8th January 2015

DOI: 10.1039/c4ra12524j

www.rsc.org/advances

\section{Usage of different vessel models in a flow-through cell: in vitro study of a novel coated balloon catheter}

\author{
Jenny Bandomir, ${ }^{a}$ Sebastian Kaule, ${ }^{\mathrm{b}}$ Klaus-Peter Schmitz, ${ }^{\mathrm{b}}$ Katrin Sternberg, ${ }^{\mathrm{b}}$ \\ Svea Petersen ${ }^{\mathrm{b}}$ and Udo Kragl ${ }^{\star a}$
}

Drug-coated balloon catheters are a novel clinical treatment alternative for coronary and peripheral artery diseases. Calcium alginate, poly(vinylethylimidazolium bromide) and polyacrylamide hydrogels were used as vessel models in this in vitro study. In comparison to a simple silicone tube their properties can be easily modified simulating different types of tissue. Local drug delivery after balloon dilation in the first crucial minute was determined in a vessel-simulating flow-through cell by a simulated blood stream. Balloon catheters were coated with paclitaxel using the ionic liquid cetylpyridinium salicylate as a novel carrier. Drug transfer from coated balloon catheters to different simulated vessel walls was evaluated and compared to a silicone tube. The highest paclitaxel delivery upon dilation was achieved with calcium alginate as the vessel model $(60 \%)$ compared to polyacrylamide with $20 \%$ drug transfer. The silicone tube showed the least amount of wash-off $(<1 \%)$ by a simulated blood stream after one minute from the vessel wall. The vessel-simulating flow-through cell was combined with a model coronary artery pathway to estimate drug loss during simulated use in an in vitro model. Calcium alginate and polyacrylamide hydrogels were used as tissue models for the simulated anatomic implantation process. In both cases, similar transfer rates for paclitaxel upon dilation were detected.

\section{Introduction}

Drug-coated balloon (DCB) technologies have emerged as a potential alternative to drug eluting stents (DES) to minimize restenosis. ${ }^{1}$ The applied drug should exhibit specific chemical properties and mechanism of action as well as pharmacokinetics and a fast transfer to be quickly absorbed by the vessel wall. ${ }^{2}$ Paclitaxel (PTX), a cytotoxic agent, was determined as the primary drug for DCB due to its efficient uptake as well as its extended retention. ${ }^{3}$ The cytotoxic, anti-proliferative effect of DES on the vessel wall has been widely explored..$^{2,4}$ Preclinical studies with DCB have shown that $3 \mu \mathrm{g} \mathrm{mm}{ }^{-2}$ paclitaxel is the effective dose to achieve an efficient, long-term, antiproliferative effect on the vessel wall., ${ }^{2,5}$ Drug delivery during angioplasty depends on drug dose, transfer system, dilation time, release pattern and appropriate balloon coating. Different balloon coating technologies are described in the literature. , $^{2,3,6-8}$ In addition to pure PTX balloon catheters different PTX formulations with additives such as urea, butyryl-tri-hexyl citrate (BTHC), iopromide and Shellac (aleuritic and shellolic acid) are commercially available. ${ }^{6}$ Kleber et al. summarized

${ }^{a}$ University of Rostock, Department of Chemistry, Albert-Einstein-Straße 3a, 18059 Rostock, Germany. E-mail: udo.kragl@uni-rostock.de

${ }^{b}$ University of Rostock, Institute for Biomedical Engineering, Friedrich-BarnewitzStraße 4, 18119 Rostock, Germany clinical evidence for these different DCBs in coronaries arteries with CE-mark (Conformité Européene). ${ }^{7}$

The Paccocath technology with PTX embedded in hydrophilic iopromide coating increases the solubility and thus the transfer of PTX to the vessel wall. More than $80 \%$ of the drug is retained during balloon implantation to the target tissue (lesion) and $10-15 \%$ of the drug is released in the vessel wall upon $60 \mathrm{~s}$ balloon inflation. ${ }^{3}$ FreePac technology uses the natural additive urea as a carrier, which should enhance drug release as well as absorption, and thereby reduce total drug elution times (30-60 s). During balloon inflation, the blood flow in the vessel is interrupted and therefore expansion can only be maintained up to one minute. Microporous balloon surfaces with Shellac coating technology can be inflated up to one minute and achieve total drug release. A shorter dilation time results in partial drug release. ${ }^{2,3}$ In a porcine model vessel wall, Scheller et al. have demonstrated a drug release of approx. 90\% after one minute inflation and 40 to 60 minutes later, they could detect about $10 \%$ PTX in the vessel wall. Thus, PTX is transferred into and retained by the pig tissue for a certain time. ${ }^{9}$

To date, there are only a few in vitro studies presented, characterizing and describing the simulated use of drug coated balloon catheters in an in vitro vessel model. ${ }^{10-12}$ The previously used models are far from physiological properties of the material, e.g. a silicone tube acts like an artery. We have been working on polymerized ionic liquids (PILs) which are able to 
form hydrogels. Depending on the type of ionic liquid and degree of cross-linking the mechanical properties can be modified. ${ }^{13}$ In our presented study, these hydrogels were evaluated to act as vessel model and compared to known hydrogels. Next to calcium alginate as a natural hydrogel, synthetic polymers with good mechanical and long-term stability were also used. ${ }^{13}$ PTX-coated balloon catheters using the ionic liquid cetylpyridinium salicylate (Cetpyrsal) as a novel innovative additive were studied. ${ }^{\mathbf{1 1}}$ Local drug delivery within a vesselsimulating flow-through cell under physiological conditions during the first crucial minute was investigated. For an assessment of this study, the total drug delivery upon dilation (retention into the hydrogel and wash-off (release) from the hydrogel compartment by a simulated blood stream) and the residual load on the balloon were analyzed. Furthermore, the drug loss during a simulated insertion was estimated by combining the flow-through cell with a model coronary artery pathway.

\section{Materials and methods}

\subsection{Materials}

Paclitaxel (PTX, $\geq 99.5 \%$ ) was obtained from Cfm Oskar Tropitzsch e.K., Germany. Sodium alginate was purchased from Fagron $\mathrm{GmbH}$ \& $\mathrm{Co} \mathrm{KG}$, Germany. Calcium sulfate dihydrate ( $\geq 99 \%$; Merck KGaA, Germany) and trisodium phosphate decahydrate ( $\geq 99 \%$; Merck KGaA, Germany) were used as received. 1-Vinylimidazole ( $\geq 99 \%$; Sigma-Aldrich, Germany), ethyl bromide ( $\geq 99 \%$; Merck KGaA, Germany), cetylpyridinium chloride (Cetpyr, $\geq 96 \%$; AppliChem GmbH, Germany) and sodium salicylate ( $\geq 99.5 \%$; Merck KGaA, Germany) were also used as received. Ammonium peroxydisulphate (APS, $\geq 98 \%$ ), tetramethylethylenediamine (TEMED, 99\%), Rotiphorese ${ }^{\circledR}$ Gel 30 (acrylamide/ bisacrylamide, $37.5: 1$ ) and Rotiphorese ${ }^{\circledR}$ Gel B (2\% bisacrylamide) were obtained from Carl Roth, Germany and used as received. Angioplasty balloon catheters of $4 \mathrm{~mm}$ in diameter and 20 or $30 \mathrm{~mm}$ length were kindly supplied by Biotronik SE \& Co KG, Germany. Ionic liquid (IL) cetylpyridinium salicylate (Cetpyrsal) was synthesized by reaction of cetylpyridinium chloride with sodium salicylate according to published procedures..11,14

\subsection{Balloon coating}

A pipetting technique was used for the coating of the inflated balloon catheter according to Petersen et al. ${ }^{11}$ Briefly, PTX and Cetpyrsal were separately dissolved in methanol to yield concentrations of $4.72 \mathrm{mg} \mathrm{mL} \mathrm{mL}^{-1}$ (both stock solutions). Following this, a Cetpyrsal-PTX solution $(50 \%, \mathrm{w} / \mathrm{w})$ was mixed from both stock solutions. $100 \mu \mathrm{L}$ of the Cetpyrsal-PTX solution was then slowly pipetted onto each balloon catheter, resulting

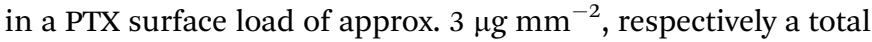
of $659.73 \mu \mathrm{g}$ (balloon 1: $3.5 \mathrm{~mm}$ diameter, $20 \mathrm{~mm}$ length), $753.99 \mu \mathrm{g}$ (balloon 2: $4.0 \mathrm{~mm}$ diameter, $20 \mathrm{~mm}$ length) or $1130.97 \mu \mathrm{g}$ (balloon 3: $4.0 \mathrm{~mm}$ diameter, $30 \mathrm{~mm}$ length). During the pipetting process, the balloon was rotated and evaporation of methanol was ensured by a gentle stream of air. Finally, all balloon catheters were dried at $23 \pm 2{ }^{\circ} \mathrm{C}$ overnight. ${ }^{11}$
Used balloons during in vitro study. Silicone tube (only balloon type 2), calcium alginate (trial 1: balloon 2, trial 2-6: balloon type 1), poly(VEImBr) hydrogel (only balloon type 2), PAAm (trial 1-3: balloon type 2, trial 4-6: balloon type 3). For the model ASTM F2394-07 only balloon type 2 was used.

\subsection{Hydrogel preparation}

Calcium alginate hydrogel. Sodium alginate $(3 \%, \mathrm{w} / \mathrm{w})$ was dissolved in de-ionized water. To $0.15 \mathrm{~g} \mathrm{CaSO}_{4} \cdot 2 \mathrm{H}_{2} \mathrm{O} 1500 \mu \mathrm{L}$ de-ionized water was added and to the resulting suspension, $500 \mu \mathrm{L}$ of a $10 \%(\mathrm{w}) \mathrm{Na}_{3} \mathrm{PO}_{4} \cdot 12 \mathrm{H}_{2} \mathrm{O}$ solution was added. $16.5 \mathrm{~g}$ alginate sol $(3 \%, \mathrm{w} / \mathrm{w})$ was mixed by a sheet of strong paper with this fresh calcium-containing suspension and was filled in the vessel-simulating flow-through cell. After complete gelation, the metal rod was removed from the flow-through cell and a simulated artificial vessel wall was obtained.

Polyacrylamide (PAAm) hydrogel. PAAm was synthesized by radical polymerization. Rotiphorese ${ }^{\circledR}$ Gel $30(3.975 \mathrm{~mL})$ was added to $10.794 \mathrm{~mL}$ de-ionized water. Polymerization was initiated by adding $210 \mu \mathrm{L}$ fresh APS solution $(10 \%, \mathrm{w} / \mathrm{w})$ and TEMED $(21 \mu \mathrm{L})$. After a short reaction time $(2-3 \mathrm{~min})$, the flowthrough cell containing the metal rod was filled with the polymerizing solution. Following full gelation, the metal rod was removed from the system

Poly(vinylethylimidazolium bromide) hydrogel (poly(VEImBr)). $7.5 \mathrm{~g}$ of 1-vinyl-3-ethyl-imidazolium bromide (36.93 mmol, [VEIm][Br]), synthesis previously described by Bandomir et al., was dissolved in $11.675 \mathrm{~mL}$ de-ionized water. $^{13}$ Rotiphorese ${ }^{\circledR}$ Gel B $(4.925 \mathrm{~mL}), 750 \mu \mathrm{L}$ APS solution $(10 \%, \mathrm{w} / \mathrm{w})$ and $150 \mu \mathrm{L}$ of TEMED were added. The solution was mixed using a Vortex and after a short reaction time (1-2 min), the flow-through cell containing the metal rod was filled with the polymerizing solution. ${ }^{13}$ The metal rod was removed following complete hardening of the hydrogel.

\subsection{Simulated use of DCB in a flow-through cell in different vessel models}

An adapted vessel-simulating flow-through cell was chosen, which is described in detail by Seidlitz et al. ${ }^{15}$ Instead of the acrylic glass disc a metal disc was used. Calcium alginate, PAAm and poly(VEImBr) hydrogels were inserted as hydrogel compartments. The DCB was placed in the simulated vessel wall and dilated for $60 \mathrm{~s}$ with a nominal pressure of 7 bar. The flowthrough cell with the inflated balloon catheter is shown in Fig. 1 with PAAm hydrogel as the vessel model. After expansion, the balloon was removed and isotonic sodium chloride $(\mathrm{NaCl}$, $0.9 \%$ ) as a perfusion medium was circulated along the simulated vessel wall for a duration of $1 \mathrm{~min}$ at a flow rate of $35 \mathrm{~mL}$ $\min ^{-1}$. Pumping of medium was managed with a gear pump (Ismatec MCP-Z ISM 405A, pump head model 186-000, Germany; Tygon ${ }^{\circledR}$ tube R 3607, 3.17 mm ID, VWR International $\mathrm{GmbH}$, Germany) and the set flow rate was adjusted to the blood flow velocity in coronaries. ${ }^{\mathbf{1 6}}$ The isotonic solution was collected in a falcon vessel and the PTX concentrations were measured by HPLC (see HPLC parameters). The process of balloon angioplasty was simulated applying an in vitro model, 

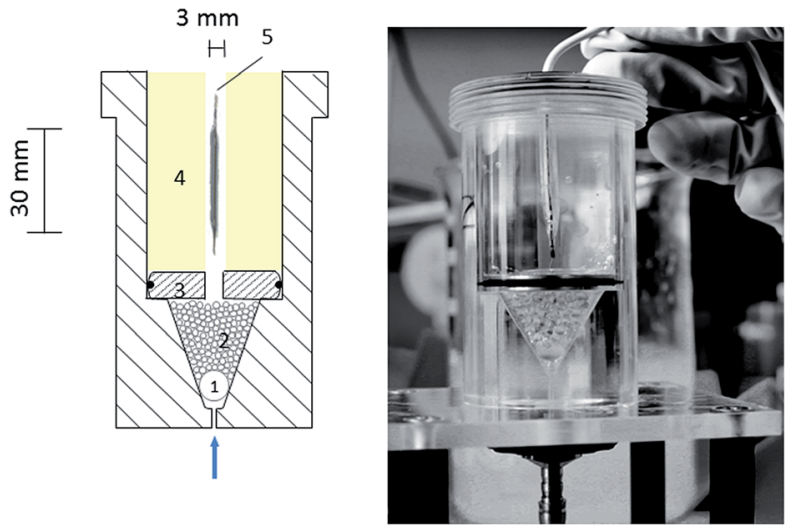

Fig. 1 Schematic drawing of the flow through cell; 1 large glass bead, 2 small glass beads, 3 metal disc, 4 hydrogel matrix, 5 drug eluting ballon (DEB).

consisting of a guiding catheter (Cordis ${ }^{\circledR}$ Vista Brite Tip ${ }^{\circledR}$; $6 \mathrm{~F}$; $1.75 \mathrm{~mm} \mathrm{ID}$; $90 \mathrm{~cm}$ ) with a guide wire (Biotronik SE \& Co KG, Galeo M 014) and a flow-through cell with different hydrogel compartments at the end of the test path, representing the vessel wall. Experiments were also performed with a silicone tube (3.0 mm ID) as the vessel model to compare the results. Paclitaxel transfer into different simulated vessel walls was measured. Before balloon dilation, the guiding catheter and vessel model were flushed with $20 \mathrm{~mL} \mathrm{NaCl-solution} \mathrm{(0.9 \% ).}$ PTX-coated balloon catheters using Cetpyrsal additive were inserted into the guiding catheter and via a guide wire, the balloon catheter was placed in the simulated vessel wall.

After balloon deflation, the pump was started (flow rate 35 $\mathrm{mL} \min ^{-1}$ ). The PTX concentration simulating drug wash-off from the vessel model within the first crucial minute was determined by HPLC measurements. The guiding catheter was then flushed with $20 \mathrm{~mL}$ methanol. The balloon catheter was extracted in $10 \mathrm{~mL}$ methanol for $30 \mathrm{~min}$ at $23 \pm 2{ }^{\circ} \mathrm{C}$ and then the residue on the balloon was analyzed. The used hydrogel after cutting into small pieces was also extracted with methanol $(20 \mathrm{~mL})$ for $30 \mathrm{~min}$ at $23 \pm 2{ }^{\circ} \mathrm{C}$ to detect the amount of transferred drug into the vessel model. The entire guiding catheter was then flushed with $20 \mathrm{~mL}$ of $0.9 \% \mathrm{NaCl}$-solution in preparation of the next experiment. In summary, the total PTX delivery upon dilation composed of drug transfer into the hydrogel and drug wash-off from the hydrogel compartment after $1 \mathrm{~min}$ by a simulated blood stream. All samples were quantified by means of HPLC after a $1: 2$ dilution with methanol.

\subsection{Simulated use of DCB in the vessel-simulating flow- through cell after passage through an in vitro vessel model according to ASTM F2394-07}

A standard anatomic model adapted from ASTM F2394-07, recently described in the literature as a standard procedure, was applied to simulate the implantation process of DCB. ${ }^{17}$ The model consisted of polymethacrylate plates forming a simulated course of a coronary artery. The used guiding catheter (Cordis ${ }^{\circledR}$ Vista Brite Tip ${ }^{\circledR} ; 6 \mathrm{~F} ; 1.75 \mathrm{~mm}$ ID; $90 \mathrm{~cm}$ ) with a guide wire (Biotronik SE \& Co KG, Galeo M 014) and the tortuous path

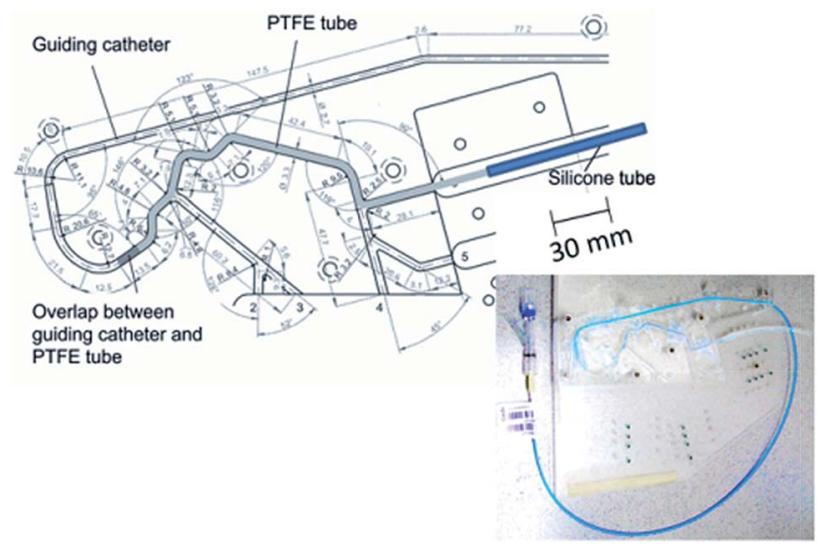

Fig. 2 Model coronary artery pathway.

equipped with a PTFE tube was placed in a $37 \pm 2{ }^{\circ} \mathrm{C}$ heated water bath (Fig. 2). The model was flushed with $30 \mathrm{~mL} 0.9 \%$ NaCl-solution. A DCB was introduced into the guiding catheter of the model and initially placed at the end of the PTFE tube. The guiding catheter was then flushed with $30 \mathrm{~mL} 0.9 \% \mathrm{NaCl}-$ solution to recover particles and PTX released during tracking. At the distal end of the test path, a hydrogel vessel model (calcium alginate or PAAm) was placed and the balloon was dilated to 7 bar and held for $1 \mathrm{~min}$. The balloon was removed after deflation and extracted in $20 \mathrm{~mL}$ methanol for 10 min (residual PTX load on the balloon) at $23 \pm 2{ }^{\circ} \mathrm{C}$. The pump was then started (flow rate $35 \mathrm{~mL} \mathrm{~min}^{-1}$ ) and the PTX concentration simulating the drug wash-off in the first crucial minute was determined. Then the used hydrogel after cutting was also extracted with methanol $(20 \mathrm{~mL})$ for $30 \mathrm{~min}$ at $23 \pm 2{ }^{\circ} \mathrm{C}$ (drug transfer into the vessel model). After balloon extraction (10 $\mathrm{min})$ in methanol, the balloon was removed and the entire pathway was then finally flushed with $30 \mathrm{~mL}$ methanol. Subsequently, the test path was flushed with $0.9 \% \mathrm{NaCl}$-solution in preparation of the next balloon dilation.

The total PTX delivery upon dilation composed of drug transfer into the vessel model (hydrogel) and drug wash-off from the hydrogel compartment after $1 \mathrm{~min}$ by a simulated blood stream. All samples were quantified by means of HPLC after a $1: 2$ dilution with methanol.

HPLC analysis. Column Eurospher C18, $120 \times 4 \mathrm{~mm} \mathrm{ID;}$ Knauer, Germany, column temperature $23{ }^{\circ} \mathrm{C}$, isocratic eluent PBS (5 mM, pH 3.5) - acetonitrile 50-50\% (v/v), flow rate $1.0 \mathrm{~mL}$ min $^{-1}$, sample volume $20 \mu \mathrm{L}$ and UV detection at $230 \mathrm{~nm}$ with calibrated measurement range $\left(0.5-20.0 \mathrm{mg} \mathrm{L}^{-1}\right) .{ }^{11}$

Particle quantification. Micro particles $(>10 \mu \mathrm{m}$ and $>25 \mu \mathrm{m})$ were quantified according to USP 788, Eur. Pharmacopeia via the particle counter HIAC ROYCO 9703 device (sensor model HRDL400, HACH, Loveland, Colorado, USA).

\section{Results and discussion}

\subsection{Comparison of different hydrogels in the flow-through cell}

The first set of experiments of DCB compared different hydrogels as tissue models to evaluate drug release of PTX. Drug 
transfer, the retention of PTX into three different hydrogels as tissue models respectively vessel walls as well as the wash-off (release) from the hydrogel compartment within a vesselsimulating flow-through cell were investigated during balloon dilation. A PTX transfer should be examined by using different hydrogel compartments to determine the influence of the tissue model relating to the PTX transfer upon dilation. Certain properties of the used hydrogels to simulate a vessel wall such as permeability, flexibility and long-term stability of synthetic polymers (poly(VEImBr) and PAAm) are of particular importance. Calcium alginate as a natural polymer is easily accessible but has limited long-term stability. Monovalent cations such as $\mathrm{Na}^{+}$dissolve the network within short time. In addition, alginate hydrogels are prone to microbial contamination. Results for various vessel models are depicted in Fig. 3. The total PTX delivery upon dilation composed of drug transfer into the hydrogel and drug wash-off from the hydrogel compartment after $1 \mathrm{~min}$ by a simulated blood stream. In the following the results from the balloon dilations will be discussed.

Drug transfer into the vessel model (Fig. 3, entry 1). The PTX transfer into the vessel models are listed in Table 1. Drug delivered in the silicone tube was extracted with methanol (38.6 $\pm 3.4 \%, 1.02 \pm 0.03 \mu \mathrm{g} \mathrm{mm}{ }^{-2}$ ). Considerably lower PTX was delivered into hydrogel-based vessel models. In the case of calcium alginate as the vessel wall, a PTX content in the hydrogel of $21.4 \pm 10.7 \%\left(0.53 \pm 0.23 \mu \mathrm{g} \mathrm{mm^{-2 }}\right)$ was detected. Alternatively, with PAAm as the vessel wall, only $2.8 \pm 1.8 \%$ $\left(<0.1 \mu \mathrm{g} \mathrm{mm}^{-2}\right)$ of PTX was transferred into the hydrogel. There are different possibilities for interpretation of the observed results. Drug transfer from coated balloons to the simulated vessel wall could occur in different ways. Paclitaxel may dissolve on contact with the hydrogel compartment and diffuse into the gel. Thus, solubility is very important for drug release and delivery. Dissolution depends on solubility of the used drug in $0.9 \%$ NaCl-solution. Liggins et al. published a maximum solubility of anhydrous PTX of $3.59 \pm 0.41 \mu \mathrm{g} \mathrm{mL} \mathrm{m}^{-1}$ in water after $3 \mathrm{~h}$ at $37^{\circ} \mathrm{C}^{18}$ Another report described a solubility of $<0.1 \mu \mathrm{g} \mathrm{mL} \mathrm{L}^{-1}$

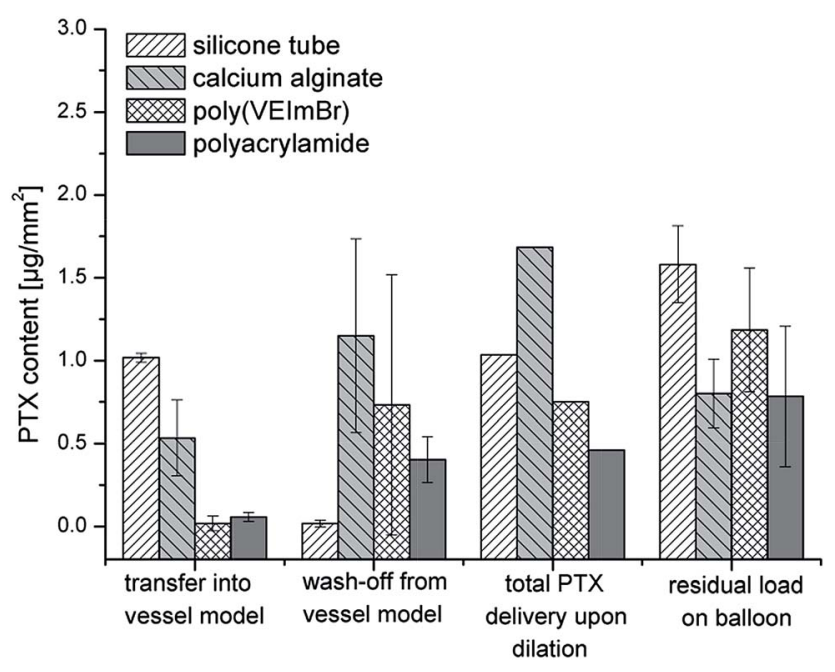

Fig. 3 Drug transfer rate for PTX in different in vitro vessel models.
Table 1 Total PTX delivery upon dilation in different vessel models after simulated use in an in vitro vessel model $^{a}$

\begin{tabular}{llll}
\hline & $\begin{array}{l}\text { Wash-off } \\
\text { after 1 min }\end{array}$ & $\begin{array}{l}\text { Transfer into } \\
\text { vessel model }\end{array}$ & $\begin{array}{l}\text { Total PTX delivery } \\
\text { upon delivery }\end{array}$ \\
\hline Silicone tube & $<1 \%$ & $38.6 \pm 3.4 \%$ & About $40 \%$ \\
Calcium alginate & $41.2 \pm 14.2 \%$ & $21.4 \pm 10.7 \%$ & About $60 \%$ \\
Poly(VEImBr) & $28.7 \pm 26.2 \%$ & $<2 \%$ & n. a. \\
PAAm & $17.8 \pm 5.3 \%$ & $2.8 \pm 1.8 \%$ & About $20 \%$ \\
a n. a.: not available. & & \\
\end{tabular}

in aqueous medium, which is quite low. ${ }^{19}$ Water solubility could be increased by synthesis of derivatives, under the risk of changing pharmaceutical characteristics. ${ }^{20}$ Due to very poor solubility of PTX in water, transport via dissolution and diffusion into the hydrogel is not responsible for the main transfer. Another drug transfer pathway may occur by particle transfer of PTX due to prevailing mechanical forces during balloon expansion onto the vessel wall. Over a period of one minute, a contact between the expanded balloon and simulated vessel wall is established, thus allowing transfer of PTX particles. The contact time was consistent in every case, but the inner diameter of the silicone tube $(3.0 \mathrm{~mm})$ was different in comparison to the artificial vessel walls $(3.14 \mathrm{~mm})$. Hence, the prevailing mechanical forces during balloon expansion in the silicone tube were stronger and more PTX could be transferred. To conclude, the main PTX transfer during balloon expansion occurred due to prevailing mechanical forces.

Furthermore, the hydrogel characteristics were important for PTX transfer and diffusion into hydrogels. ${ }^{21}$ PAAm and poly(VEImBr) were synthetic polymers with a specific cross-linker content (poly(VEImBr): $1.7 \%$ to PAAm: $0.8 \%$ cross-linker content). ${ }^{13}$ On the contrary, the calcium alginate hydrogel is a natural polymer with variability in its properties. In addition to mechanical properties (flexibility) of the vessel models, different adhesion properties were present. This corresponds to different amounts of PTX wash-off from the vessel models after 1 min by a simulated blood stream (see Table 1 or Fig. 3). Moreover, the diffusion of PTX into the vessel wall occurs at various rates, which may be related with the cross-linker content. This leads to PTX diffusion into synthetic polymers < $5 \%$ (poly(VEImBr) and PAAm) compared to the natural polymer of $21.4 \pm 10.7 \%$.

Drug wash-off from various vessel models after $1 \mathrm{~min}$ (Fig. 3, entry 2). A drug release time of only one minute was chosen to simulate a very fast PTX transfer and wash-off from the vessel model. The silicone tube is a hydrophobic material and showed the least amount of wash-off $(<1 \%)$ from the vessel model (see Table 1). Silicone tubes as a vessel model were not very suitable because they are not similar to physiological uptake behavior. A hydrogel is a network of polymer chains that are hydrophilic and should be more appropriate. ${ }^{22,23}$ With a hydrogel compartment as a vessel wall, the PAAm was able to achieve the lowest wash-off quantities $\left(17.8 \pm 5.3 \%, 0.40 \pm 0.14 \mu \mathrm{g} \mathrm{mm}^{-2}\right)$, compared to poly(VEImBr) $(28.7 \pm 26.2 \%)$ and calcium alginate 
$\left(41.2 \pm 14.2 \%, 1.15 \pm 0.58 \mu \mathrm{g} \mathrm{mm}^{-2}\right)$. Thus, the highest drug wash-off after 1 min was achieved in case of calcium alginate as the vessel model. The simulated vessel models chosen were important for an effective drug transfer. Thus, the drug delivery characteristic is dependent on the hydrogel compartment. With poly(VEImBr) as the hydrogel compartment, some analytical problems occurred. Thus, their potential could not be fully explored. The poly(VEImBr) hydrogel shows strong swelling behavior in methanol which was used to extract the drug from the hydrogel. Most of the solvent diffused into the polymer and thus the hydrogel rapidly swells. In addition, the high salinity compromised the HPLC analysis of PTX (value for drug washoff, see Table 1) and therefore elongated peaks in the chromatogram were difficult to integrate together with a low interpretable reproducibility of the data. This could be overcome by using other drug candidates or models showing, for example, fluorescence. In summary, the hydrogel material was crucial for the total drug delivery upon dilation (Fig. 3). Since the drug is poorly soluble in water and because of binding to tissue structures, the PTX may persist longer in the vessel wall. Calculated curves for PTX tissue concentration as function of time are provided in the literature. Within the first hour, the concentration decreases dramatically. ${ }^{24}$

Drug residue on the balloon. The residual loads of PTX on the balloon catheter were also determined (Fig. 3, entry 4). Extraction of the balloon in methanol resulted in the highest PTX concentration for the silicone tube $(59.5 \pm 4.6 \%, 1.6 \pm 0.2$ $\mu \mathrm{g} \mathrm{mm}^{-2}$ ) as the vessel model, meaning most of the PTX remained on the balloon surface. Only $40 \%$ of the drug could be transferred during balloon dilation. However, considerably less drug on the balloon catheter surface were analyzed in the cases of dilation in calcium alginate $\left(30.8 \pm 7.6 \%, 0.8 \pm 0.2 \mu \mathrm{g} \mathrm{mm} \mathrm{mm}^{-2}\right)$ and PAAm $\left(33.2 \pm 15.3 \%, 0.8 \pm 0.4 \mu \mathrm{g} \mathrm{mm}^{-2}\right)$ as vessel models. Consequently, in both cases about $70 \%$ of the drug is removed from the balloon catheter.

As already mentioned, PTX is characterized by its very low solubility. The balloon catheters used here exhibit homogeneous coating due to the use of an IL as a novel additive (Cetpyrsal/PTX, 50/50, w/w). There are no needle-like crystals present on the balloon surface. ${ }^{11}$ Previous experiments showed that the novel additive reduced the drug loss compared to a commercially available DCB with an urea-based coating. ${ }^{11}$ For this reason, there is the possibility to deliver (transfer) more PTX during the balloon expansion and therefore we concentrated on this novel DCB. The degree of crystallization is important; Afari et al. published that more crystalline coatings yield higher tissue levels and biological efficacy. ${ }^{25}$ In contrast, less crystalline coatings resulted in improved uniformity and less particle formation. ${ }^{25}$ Heilmann et al. had found (via an in vivo study) that the advantageous effect of a hydrophilic additive such as using iopromide for higher tissue concentrations was antagonized by increased amounts of wash-off of used coatings. $^{26}$

Drug loss is a process constituted of mechanical loss by sheath passage and collisions with the vessel wall and dissolution of the coating in the blood stream. ${ }^{26}$ This process will be simulated using a standard anatomic model adapted from
ASTM F2394-07 (described in next section). Drug adherence and loss on the way to the vessel was tested in vitro by Kelsch et al. ${ }^{\mathbf{8}}$ Drug loss upon passage through a blood-filled hemostatic valve and guiding catheter for one minute in stirred blood at $37{ }^{\circ} \mathrm{C}$ was investigated. Urea-based DCB lost $26 \pm 3 \%$ and iopromidebased DCB lost $36 \pm 11 \%$ of the total amount on the balloon. ${ }^{8}$

In conclusion for the simulated use of DCB, the total drug delivery upon dilation is different for the used hydrogels simulating the vessel wall. Calcium alginate hydrogel as the vessel model showed the highest PTX delivery upon dilation. The wash-off from the alginate hydrogel was high (drug release after $1 \mathrm{~min}$ by a simulated blood stream: $41.2 \pm 14.2 \%$ ). However, $21.4 \pm 10.7 \%$ of the drug diffused into the hydrogel compartment. The silicone tube showed the least amount of wash-off $(<1 \%)$ from the vessel model after $1 \mathrm{~min}$, but it is quite different to natural vessels. Poly(VEImBr) hydrogels as vessel models were difficult to analyze. In the case of PAAm as the vessel model, only $20 \%$ of PTX could be delivered upon dilation.

\subsection{Simulated use of DCB in the vessel-simulating flow- through cell after passage through an in vitro vessel model according to ASTM F2394-07}

In order to simulate the implantation process, the vesselsimulating flow-through cell was combined with a model coronary artery pathway to estimate drug loss and transfer as well as particle release. Cetpyrsal-based DCBs were manually advanced through a tortuous vessel path, consisting of a guiding catheter with a guide wire. Calcium alginate and polyacrylamide hydrogels were used as tissue models for the simulated use in an in vitro model (Fig. 4). The obtained results can be compared with the data from Petersen et al. ${ }^{11}$ In their study, they also used the anatomic model according to ASTM F2394-07 with a silicone tube as the vessel model.

Total PTX delivery upon dilation (Fig. 4, entry 1). Only small transferred fractions were observed for both vessel models after passage of the balloon catheter through a simulated anatomic model. In the case of PAAm, a total PTX delivery upon dilation of $5.1 \pm 2.1 \%\left(0.14 \pm 0.06 \mu \mathrm{g} \mathrm{m^{-2 }}\right)$ was achieved. Similar

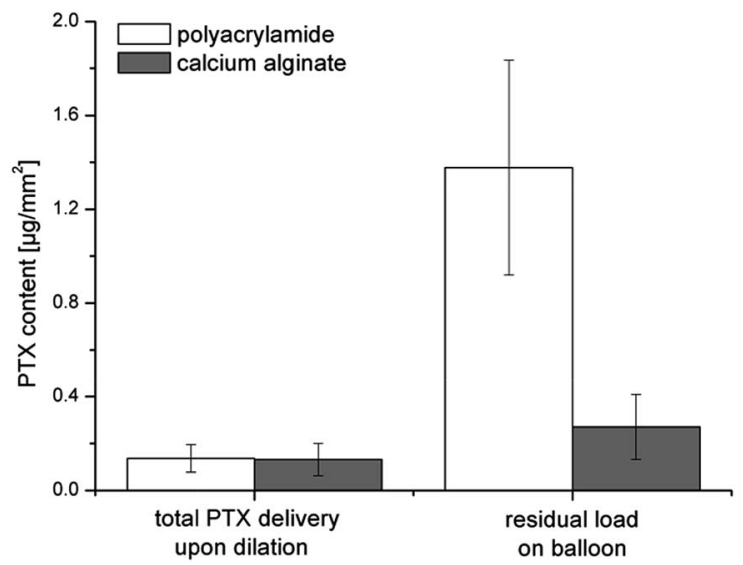

Fig. 4 Total drug transfer rate upon dilation for PTX after simulated anatomic passage. 
transfer rates for PTX upon dilation were detected with calcium alginate as the vessel model $\left(6.4 \pm 3.8 \%, 0.13 \pm 0.07 \mu \mathrm{g} \mathrm{mm}{ }^{-2}\right)$. As before, a short wash-off time (drug release after one minute) was chosen to simulate the drug behavior after pass through the tracking model. With PAAm as the vessel model, a PTX content of $1.7 \pm 0.7 \%$ could be detected in the wash-off solution. A similar value for calcium alginate as the vessel model was found (PTX content of $2.0 \pm 1.1 \%$ ) as wash-off from the hydrogel compartment in the first minute. The PTX transfer into the hydrogel compartment was slightly higher (PAAm: of $3.4 \pm$ $1.9 \%$; calcium alginate: of $4.3 \pm 2.8 \%$ ). Thus, the drug diffused into the vessel model or adhered on the vessel wall and was not released in one minute into the medium. However, the total PTX delivery upon dilation was similar for two different vessel models after the simulated implantation process. Petersen et al. transferred more PTX in the silicone tube (up to $40 \%$ ) with a PTX-Cetpyrsal balloon catheter $(50: 50, \mathrm{w} / \mathrm{w})$ coated in a folded condition. With the balloon coated in an expanded condition, the PTX transfer in the silicone tube was lower $(5-15 \%) .{ }^{11}$ Here, the used balloon catheters were coated in an expanded condition. Seidlitz et al. used pure PTX-coated balloons and showed PTX transfer rates to gel below $1 \%$ (calcium alginate as vessel model) ${ }^{12}$ In their study, they also used a model of a coronary artery pathway to investigate drug loss and drug transfer to the gel. However, in our study with the novel DCB coating more PTX was delivered upon dilation (calcium alginate: $6.4 \pm 3.8 \%$ compared to below 1\%). In conclusion, the PTX transfer upon dilation depends on the coating of the balloon and the used vessel model simulating the vessel wall.

Drug residue on the balloon (Fig. 4, entry 2). Extraction of the balloon catheter in methanol resulted in a PTX content of $1.38 \pm 0.46 \mu \mathrm{g} \mathrm{mm}{ }^{-2}$ with PAAm as the vessel model (Fig. 4). Consequently, there was still $51.4 \pm 15.7 \%$ PTX remaining on the balloon surface and about $50 \%$ of the drug is removed from the balloon catheter. However, expansion of the balloon in calcium alginate yielded only $0.27 \pm 0.14 \mu \mathrm{g} \mathrm{mm} \mathrm{m}^{-2}$ PTX residue on the balloon $(13.3 \pm 8.3 \%)$. The balloon was almost completely unloaded.

Particle quantification. In addition to the total drug delivery upon dilation, particle measurements $(>10 \mu \mathrm{m},>25 \mu \mathrm{m})$ were performed after track and dilation of the balloon (Table 2). These size limits $(>10 \mu \mathrm{m},>25 \mu \mathrm{m})$ are assumed from the evaluation of surface and coating damage of stent delivery catheter. The estimated mechanism from DCB involves the delivery of particles to the inner lumen of coronary arteries, the release of particles or coating fragments in the coronary

Table 2 Particle quantification after simulated anatomic passage

\begin{tabular}{lcccc}
\hline & $\begin{array}{l}\text { PAAm } \\
>10 \mu \mathrm{m}\end{array}$ & $\begin{array}{c}\text { PAAm } \\
>25 \mu \mathrm{m}\end{array}$ & $\begin{array}{c}\text { Calcium alginate } \\
>10 \mu \mathrm{m}\end{array}$ & $\begin{array}{c}\text { Calcium alginate } \\
>25 \mu \mathrm{m}\end{array}$ \\
\hline $\begin{array}{l}\text { After track } \\
\text { After } \\
\text { expansion }\end{array}$ & $\begin{array}{c}230 \pm 126 \\
\text { Sum }\end{array}$ & $\begin{array}{c}33 \pm 16 \\
580 \pm 308\end{array}$ & $\begin{array}{c}56 \pm 27 \\
9 \pm 1\end{array}$ & $1 \pm 1$ \\
& $234 \pm 127$ & $34 \pm 17$ & $589 \pm 309$ & $57 \pm 28$
\end{tabular}

arteries. Complications are occlusions of small vessels or capillaries. ${ }^{17,27}$ Quantified particles are mainly PTX particles because Cetpyrsal does not form any ascertainable particles in aqueous solution under used conditions.

Using calcium alginate as the vessel model, a total of $589 \pm$ 309 particles $(>10 \mu \mathrm{m})$ per $\mathrm{mm}^{2}$ were analyzed. Contained particles $>25 \mu \mathrm{m}$ per $\mathrm{mm}^{2}$ were detected in a ratio of $1: 10(57 \pm$ 28). In the second test series using PAAm, the expected sum of particles was decreased $\left(234 \pm 127(>10 \mu \mathrm{m})\right.$ per $\mathrm{mm}^{2}, 34 \pm 7$ $(>25 \mu \mathrm{m})$ per $\mathrm{mm}^{2}$ balloon surface). Petersen et al. described that DCB based on Cetpyrsal generated a lower quantity of particles (expanded condition: $280 \pm 91$ particles $(>10 \mu \mathrm{m})$ per $\mathrm{mm}^{2}$ balloon surface) compared to commercially available DCB using a urea-based coating $(329 \pm 161$ particles $(>10 \mu \mathrm{m})$ per $\mathrm{mm}^{2}$ balloon surface).$^{11}$ Amounts of particles generated from the PTCA balloon catheters by comparing two modified lubricous polymeric hydrogel coatings used at various thicknesses were demonstrated by Babcock et $a l^{28}$ In their study, a submicron coating (dry thickness of $0.5 \mu \mathrm{m}$ ) generates far fewer particulates than the micron coating (dry thickness of $2 \mu \mathrm{m}$ ) on the same substrate in a standard anatomic model adapted from ASTM F2394-07. ${ }^{28}$

\section{Conclusions}

Drug-coated balloon catheters are an alternative for coronary and peripheral artery disease. Based on the limited number of published results of in vitro characterization of drug coated balloons, there is a need for further research. Novel PTX-coated balloons using ionic liquid Cetpyrsal as an additive for the in vitro study were applied. Drug delivery upon dilation in different tissue models (calcium alginate, poly(VEImBr) and PAAm) using a vessel-simulating flow-through cell was investigated and compared to a silicone tube as the tissue model. The highest PTX delivery upon dilation was achieved with calcium alginate as the vessel model (about 60\%). However, a total PTX delivery upon dilation of $20 \%$ was determined with polyacrylamide as vessel model. The used vessel models showed seemingly various adhesion properties, thus the PTX wash-off quantities during simulated blood flow were different. The silicone tube showed the lowest amount of wash-off $(<1 \%)$ from the vessel model after 1 min simulated blood stream. The highest drug wash-off (release) was achieved with calcium alginate as vessel model. Moreover, the diffusion of PTX into the vessel wall occurs at various rates, which may be related to the cross-linker content of the hydrogels. In addition to solubility and thus diffusion of PTX, the hydrogel material as well as the coating was crucial for drug transfer from the balloon into the vessel wall when compared to reported data. Furthermore, the vessel-simulating flow-through cell was combined with a model coronary artery pathway to simulate an anatomic implantation process. Vast amounts of the coated drug were lost during a simulated artery pathway. Only a small fraction of the total loads of PTX were delivered upon dilation. Similar transfer rates for PTX upon dilation were achieved with calcium alginate and PAAm as vessel models. The crucial drug delivery upon dilation was examined with the aid of different hydrogel materials to 
evaluate the in vitro research. These are important data for the in vivo application.

\section{Acknowledgements}

The authors thank Jana Unger for skillful assistance in synthesizing the ionic liquids and Dr Thomas Reske as well as Philip Wahl for their expert technical assistance. We also acknowledge Biotronik SE \& Co KG for a generous supply of the uncoated balloon catheters. For fruitful discussions within the REMEDIS network we thank the group of Prof. Dr Katrin Sternberg, IBMT University of Rostock. Furthermore, we would like to thank Dr Anne Seidlitz, Dr Beatrice Semmling and Prof. Dr Werner Weitschies for cooperation and design of the vesselsimulating flow-through cell. Financial support by Bundesministerium für Bildung und Forschung (BMBF) within REMEDIS "Höhere Lebensqualität durch neuartige Mikroimplantate" (FKZ:03IS2081) is gratefully acknowledged.

\section{References}

1 A. Lupi, A. Rognoni, G. G. Secco, I. Porto, F. Nardi, M. Lazzero, L. Rossi, R. Parisi, R. Fattori, G. Genoni, R. Rosso, P. R. Stella, I. Sheiban, L. Bolognese, F. Liistro, A. S. Bongo and P. Agostoni, Int. J. Cardiol., 2013, 168, 4608-4616.

2 M. Krokidis, S. Spiliopoulos, K. Katsanos and T. Sabharwal, Cardiovasc. Intervent. Radiol., 2013, 36, 281-291.

3 R. Waksman and R. Pakala, Circ.: Cardiovasc. Interventions, 2009, 2, 352-358.

4 I. Narbute, S. Jegere, I. Kumsars, I. Mintale, I. Zakke, K. Bumeistere, D. Sondore, A. Grave and A. Erglis, Medicina, 2011, 47, 536-543.

5 A. Posa, N. Nyolczas, R. Hemetsberger, N. Pavo, O. Petnehazy, Z. Petrasi, G. Sangiorgi and M. Gyongyosi, Cathet. Cardiovasc. Interv., 2010, 76, 395-403.

$6 \mathrm{~J}$. P. Loh and R. Waksman, JACC: Cardiovascular Interventions, 2012, 5, 1001-1012.

7 F. X. Kleber, H. Rittger, K. Bonaventura, U. Zeymer, J. Wohrle, R. Jeger, B. Levenson, S. Mobius-Winkler, L. Bruch, D. Fischer, C. Hengstenberg, T. Porner, D. Mathey and B. Scheller, Clin. Res. Cardiol., 2013, 102, 785-797.

8 B. Kelsch, B. Scheller, M. Biedermann, Y. P. Clever, S. Schaffner, D. Mahnkopf, U. Speck and B. Cremers, Invest. Radiol., 2011, 46, 255-263.

9 B. Scheller, U. Speck, C. Abramjuk, U. Bernhardt, M. Bohm and G. Nickenig, Circulation, 2004, 110, 810-814.
10 S. Petersen, I. Minrath, S. Kaule, J. Kocher, K. P. Schmitz and K. Sternberg, Coatings, 2013, 3, 253-267.

11 S. Petersen, S. Kaule, F. Stein, I. Minrath, K. P. Schmitz, U. Kragl and K. Sternberg, Mater. Sci. Eng., C, 2013, 33, 4244-4250.

12 A. Seidlitz, N. Kotzan, S. Nagel, T. Reske, N. Grabow, C. Harder, S. Petersen, K. Sternberg and W. Weitschies, PLoS One, 2013, 8, e83992.

13 J. Bandomir, A. Schulz, S. Taguchi, L. Schmitt, H. Ohno, K. Sternberg, K.-P. Schmitz and U. Kragl, Macromol. Chem. Phys., 2014, 215, 716-724.

14 K. Bica, C. Rijksen, M. Nieuwenhuyzen and R. D. Rogers, Phys. Chem. Chem. Phys., 2010, 12, 2011-2017.

15 A. Seidlitz, S. Nagel, B. Semmling, N. Grabow, H. Martin, V. Senz, C. Harder, K. Sternberg, K.-P. Schmitz, H. K. Kroemer and W. Weitschies, Eur. J. Pharm. Biopharm., 2011, 78, 36-48.

16 C. Dimario, N. Meneveau, R. Gil, P. Dejaegere, P. J. Defeyter, C. J. Slager, J. Roelandt and P. W. Serruys, Am. J. Cardiol., 1993, 71, D54-D61.

17 W. Schmidt and P. Lanzer, in Catheter-Based Cardiovascular Interventions, ed. P. Lanzer, Springer-Verlag Berlin, Heidelberg, P. Lanzer edn, 2013, pp. 445-472.

18 R. T. Liggins, W. L. Hunter and H. M. Burt, J. Pharm. Sci., 1997, 86, 1458-1463.

19 T. Konno, J. Watanabe and K. Ishihara, J. Biomed. Mater. Res., Part A, 2003, 65A, 209-214.

20 Y. L. Khmelnitsky, C. Budde, J. M. Arnold, A. Usyatinsky, D. S. Clark and J. S. Dordick, J. Am. Chem. Soc., 1997, 119, 11554-11555.

21 N. A. Peppas, B. V. Slaughter, M. A. Kanzelberger, K. Matyjaszewski and M. Möller, in Polymer Science: A Comprehensive Reference, Elsevier, Amsterdam, 2012, vol. 9, pp. 385-395.

22 Y. G. Kim, C. H. Lee and Y. C. Bae, Fluid Phase Equilib., 2014, 361, 200-207.

23 J. K. Kim, H. J. Kim, J. Y. Chung, J. H. Lee, S. B. Young and Y. H. Kim, Arch. Pharmacal Res., 2014, 37, 60-68.

24 A. Ruebben, J. Boeing and N. Weiss, J. Intervent. Cardiol., 2010, 5, 74-76.

25 M. Afari and J. Granada, Endovascular Today, 2012, 53-58.

26 T. Heilmann, C. Richter, H. Noack, S. Post, D. Mahnkopf, A. Mittag, H. Thiele and H.-R. Figulla, European Cardiology Review, 2010, 6, 40-44.

27 B. Cortese and A. Bertoletti, Int. J. Cardiol., 2012, 161, 4-12. 28 D. E. Babcock, R. W. Hergenrother, D. A. Craig, F. D. Kolodgie and R. Virmani, Biomaterials, 2013, 34, 3196-3205. 